Published online 2017 April 13.

Abstract

\title{
Cerebral Vein and Sinus Thrombosis
}

\author{
Nader Jamalzadeh ${ }^{1, *}$ \\ ${ }^{1} \mathrm{MD}$, Radiologist \\ "Corresponding author: Nader Jamalzadeh, MD, Radiologist. E-mail: naderjamalzadeh@gmail.com
}

Received 2016 December 21; Accepted 2017 February 08.

\begin{abstract}
Although cerebral venous thrombosis is an uncommon cause of stroke among the young, it is being increasingly recognised. A prothrombotic risk factor is identified in the majority of the patients. In most instances it is seen in women in the post-partum period and those on oral contraceptives. Magnetic Resonance Imaging (MRI) has improved our ability to diagnose this condition however the variability of radiological and clinical presentation remains a challenge. MR in combination with MR venography (MRV) is the single most sensitive diagnostic technique. The MR appearance of the thrombus within the dural sinus or cortical vein is variable and is largely dependent on its age. The loss of the normal flow void on spin echo T2 images is a sensitive parameter. Thrombus on MRV is seen as loss of high flow signal from the sinus. Focal parenchymal changes occur in approximately $50 \%$ of cases and are due to oedema and infarction, with or without haemorrhage. Appearances of these lesions too are dependent on their age. Diffuse changes of raised intracranial pressure with gyral effacement may also be present.
\end{abstract}

This is an abstract presented in the 33rd Iranian congress of radiology (ICR) and the 15th congress of Iranian radiographic science association (IRSA). 